\title{
CORRIGENDUM
}

\section{Thrombospondin-1 repression is mediated via distinct mechanisms in fibroblasts and epithelial cells}

RS Watnick, RK Rodriguez, S Wang, AL Blois, A Rangarajan, T Ince and RA Weinberg

Oncogene (2015) 34, 2949-2950; doi:10.1038/onc.2015.183

Correction to: Oncogene (2015) 34, 2823-2835; doi: 10.1038/ onc.2014.228; published online 11 August 2014

The authors wish to make the readers aware of two errors that were missed at proof stage.

The first is the inclusion of an actin loading blot in Figure 4c. In fact, no loading control was needed as the purpose of this figure is merely to show that the protein is expressed in both cell lines and

a

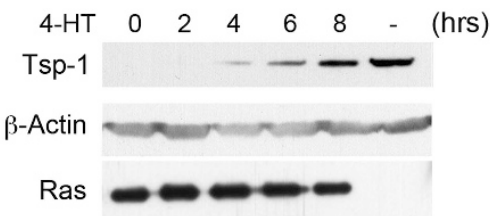

C

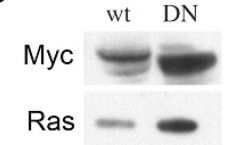

b

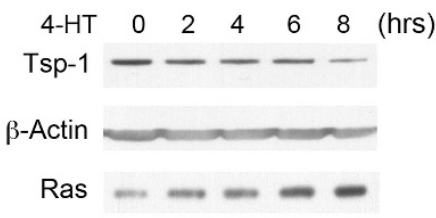

d

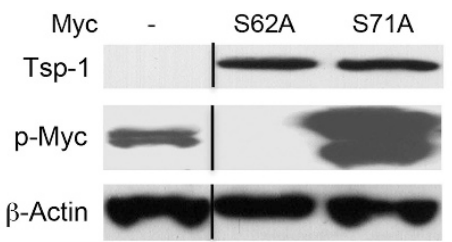

e

Myc - S62A S71A - S62A S71A

Ras low low low high high high

Tsp-1

$\beta$-Actin

Ras

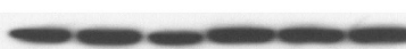

$\mathbf{f}$

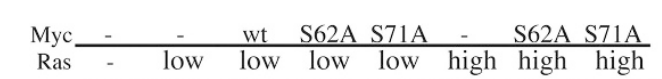

ODC

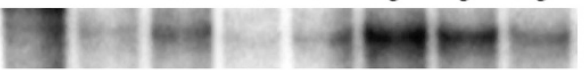

Cyclophilin not to compare its expression between the two lines. The corrected figure is shown below.

The second is with regard to Figure $5 f$. This panel was intended to be replaced but was overlooked in review.

The authors apologise for any inconvenience caused.

This error has now been rectified, and the corrected article appears in this issue. The html and online pdf versions have also been rectified, and now carry the corrected paper.

Figure 4. Effects of Myc activity on Tsp-1 expression. (a) Immunoblot analysis of Tsp-1, $\beta$-actin and Ras proteins expressed by kidney-derived cells expressing high levels of oncogenic Ras plus dominant-negative MycER after various treatment times with 4-HT. (b) Immunoblot analysis of Tsp-1, $\beta$-Actin and Ras proteins expressed by kidneyderived cells expressing low levels of oncogenic Ras plus MycER after various treatment times with 4-HT. (c) Immunoblot analysis of Myc and Ras proteins in low Ras cells expressing MycER (wt) and high Ras cells expressing DNMycER (DN). (d) Immunoblot analysis of Tsp-1, phospho Myc, $\beta$-Actin and Ras proteins expressed by kidney-derived cells expressing high levels of oncogenic Ras transfected with S62AMyc (62) or S71AMyc (71) genes. (e) Immunoblot analysis of Tsp-1, $\beta$-Actin and Ras proteins expressed by kidney-derived cells expressing low levels of oncogenic Ras, or high levels of oncogenic Ras that were mock transfected or transfected with S62AMyc (62) andS71AMyc (71) genes. (f) Ribonuclease protection assay of ODC and cyclophilin expressed in kidney-derived cells expressing no oncogenic Ras $(-)$, low levels of oncogenic Ras, or high levels of oncogenic Ras that were mock transfected or transfected with wtMyc (58), S62AMyc (62) or S71AMyc (71) genes. Black lines represent cases where lanes were digitally removed from the scanned image. 
a

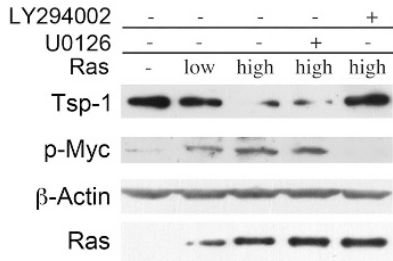

c

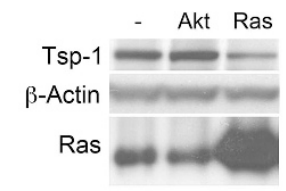

e

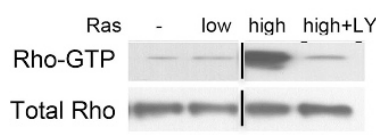

g

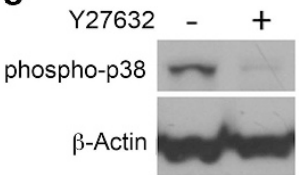

i

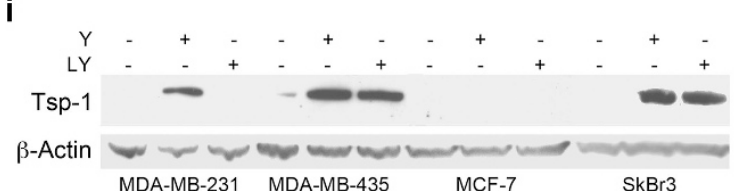

b

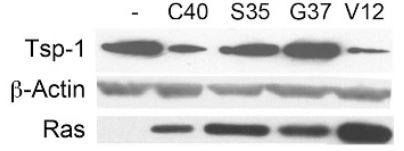

d

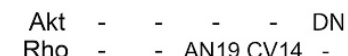

Ras low high high low high

Tsp-1 — -

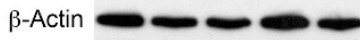

Ras

f

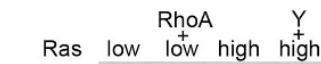

Tsp-1

p-Myc

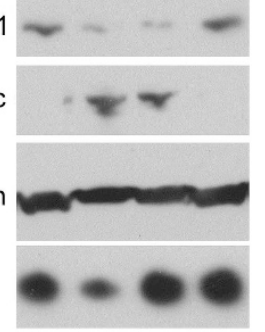

h

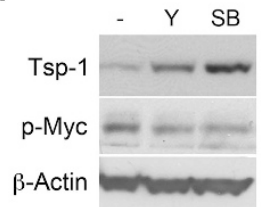

Figure 5. Effects of Ras signaling pathways on Myc phosphorylation and Tsp-1 expression. (a) Immunoblot analysis of Tsp-1, phospho Myc, phospho ERK1/2, $\beta$-Actin and Ras proteins expressed by kidney-derived cells expressing no oncogenic Ras $(-)$, low levels of oncogenic Ras or high levels of oncogenic Ras that were otherwise untreated or treated with either UO126 or LY294002. (b) Immunoblot analysis of Tsp-1, $\beta$ Actin and Ras proteins expressed by kidney-derived cells expressing no oncogenic Ras (-), RasV12C40 (C40), RasV12G37 (G37) and RasV12S35 (S35), or high levels of oncogenic Ras (V12). (c) Immunoblot analysis of Tsp-1, $\beta$-Actin and Ras proteins expressed by kidney-derived cells expressing no oncogenic Ras (-), no oncogenic Ras plus myristoylated Akt (Akt) or high levels of oncogenic Ras. (d) Immunoblot analysis of Tsp-1, $\beta$-Actin and Ras proteins expressed by kidney-derived cells expressing low levels of oncogenic Ras, low levels of oncogenic Ras plus RhoCV14 (C14), high levels of oncogenic Ras, high levels of oncogenic Ras plus RhoAN19 (AN19) and high Ras plus dominant-negative Akt (DNAkt). (e) Immunoblot analysis of GTP-bound Rho and total Rho in kidney-derived cells expressing no oncogenic Ras (-), low levels of oncogenic Ras, high levels of oncogenic Ras or high levels of oncogenic Ras plus LY294002. (f) Immunoblot analysis of Tsp-1, phospho Myc and Ras proteins expressed by kidney-derived cells expressing low levels of oncogenic Ras, low levels of oncogenic Ras plus RhoAV14 (AV14), high levels of oncogenic Ras or high levels of oncogenic Ras plus Y27632. (g) Immunoblot analysis of phospho p38MAPK and $\beta$-Actin levels in human mammary epithelial cells expressing high levels of oncogenic Ras that were untreated (-) or treated with Y27632. (h) Immunoblot analysis of Tsp-1, phospho Myc and $\beta$-Actin expressed by human mammary epithelial cells expressing high levels of oncogenic Ras that were untreated ( - ) or treated with Y27632 (Y) or SB203580 (SB). (i) Immunoblot analysis of Tsp-1 and $\beta$-actin expressed in human breast cancer cell lines treated with Y27632, LY294002. Black lines represent cases where lanes were electronically removed from the scanned image. 